MOLECULAR NEUROSCIENCE

\section{Stress hormones Trk neurons into survival}

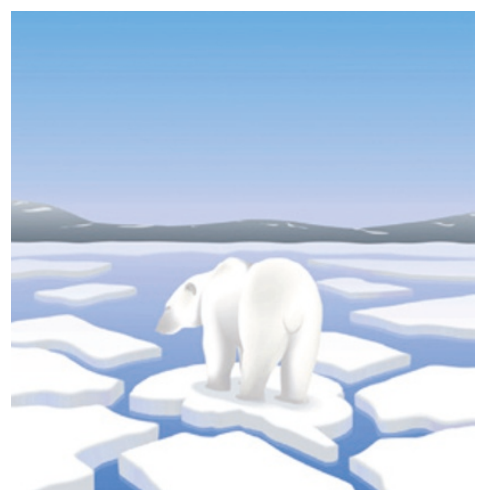

Glucocorticoids have a bad reputation. However, although these stress hormones can be neurotoxic in high levels, they are also required for neuronal survival, and they promote neuronal growth and differentiation and support synaptic plasticity in the hippocampus. Chao and colleagues now show that, in rats, the neuroprotective effects of glucocorticoids are mediated by the activation of neurotrophin receptors.

The authors showed that elevated levels of the endogenous glucocorticoid corticosterone or administration of the synthetic glucocorticoid dexamethasone (Dex) resulted in phosphorylation of the neurotrophin receptor TrkB in hippocampal cell lysates. In brain sections of Dextreated rats, phosphorylated TrkB was detected in the subgranular zone of the dentate gyrus and in the subventricular zone, two regions in which adult neurogenesis occurs. Surprisingly, Dex administration did not alter levels of the neurotrophins nerve growth factor (NGF), brainderived neurotrophic factor (BDNF) and neurotrophin 3 (NT3) in the hippocampus or in the parietal cortex, indicating that the phosphorylation of TrkB by glucocorticoids did not require increased neurotrophin production.

Phosphorylated Trks are activated tyrosine kinases, which can phosphorylate other proteins. Thus, adding Dex or BDNF (the main ligand for the TrkB receptor) to cortical slices activated TrkB and phosphorylated the intracellular signalling molecules AKT, phospholipase C $\gamma$ (PLC $\gamma$ ) and extracellular signal-regulated kinase (ERK), indicating downstream activation of the phosphatidylinositol 3-kinase (PI3K) pathway.

Because neurotrophins promote neuronal survival by binding to Trk receptors, the authors investigated whether glucocorticoid-induced activation of TrkB is also neuroprotective. They removed trophic factors from the culture medium of primary hippocampal and cortical neurons, a manipulation that normally induces apoptosis. However, treatment with Dex or BDNF rescued over $30 \%$ of neurons. Dex and BDNF probably acted through the same mechanism, as their effects were not additive.
Similar to the in vivo experiments, Dex treatment of cultured neurons did not increase levels of BDNF, NGF or NT3, suggesting that the neuroprotective effect of Dex is independent of neurotrophin release. Administration of an inhibitor of the PI3K-AKT pathway abolished Dexmediated neuroprotection, whereas adding a Trk inhibitor only reduced it; thus, glucocorticoids might also stimulate the PI3K-AKT pathway through a route that does not involve TrkB phosphorylation.

The mechanism by which glucocorticoids activate Trks is unknown but probably involves the glucocorticoid receptor, as addition of a glucocorticoid receptor antagonist abolished Dex-mediated neuroprotection. The glucocorticoid effects were slow and lasted for several hours, which is suggestive of genomic actions. Indeed, Trk activation by Dex could be abolished by actinomycin D and cycloheximine, inhibitors of transcription and translation, respectively.

This study raises some interesting questions. For example, which genes are upregulated by glucocorticoids, with Trk phosphorylation as a result? And do glucocorticoids also have neuroprotective effects in vivo? If they do, it will be interesting to investigate why glucocorticoids are associated with neurotoxicity in models of neuron death such as experimental hypoxia/ischaemia.

Leonie Welberg

ORIGINAL RESEARCH PAPER Jeanneteau, F., Garabedian, M. J. \& Chao, M. V. Activation of Trk neurotrophin receptors by glucocorticoids provides a neuroprotective effect. Proc. Natl Acad. Sci. USA 105, 4862-4867 (2008) 